\title{
Science of Circadian Rhythms
}

Editor

PHYLLIS C. ZEE

\section{SLEEP MEDICINE CLINICS}

www.sleep.theclinics.com

Consulting Editor

TEOFILO LEE-CHIONG Jr

December 2015 • Volume 10 • Number 4 